\title{
In DAA We Trust: Key Factors Essential to HCV Elimination
}

\author{
Mary Jane Burton ${ }^{1}$
}

Published online: 6 August 2019

(c) Springer Science+Business Media, LLC, part of Springer Nature 2019

The World Health Organization has issued a call for global elimination of viral hepatitis as a public health threat by 2030. To meet hepatitis $\mathrm{C}$ virus (HCV) elimination targets in the USA, a consensus committee of the National Academies of Science and Medicine (NASEM) proposes, in addition to expanded HCV screening and prevention efforts, unrestricted access to HCV direct-acting antivirals (DAAs) and aggressive treatment of estimated 260,000 unique patients annually [1]. Sustaining this level of treatment of HCV will require removal of multiple barriers at the system and at the provider and patient levels. The NASEM report gives specific recommendations for terminating system-level barriers regarding DAA access. Using new venues for $\mathrm{HCV}$ treatment, including primary care clinics and penitentiaries, will increase access to HCV prescribers. Removing health plan restrictions based on fibrosis level or active substance use disorder (SUD) will also promote DAA treatment. To fully achieve universal HCV treatment, impediments at the provider and patient levels must also be circumvented.

Researchers affiliated with the Veterans Affairs (VA) Pittsburgh Healthcare System's Center for Health Equity Research and Promotion (CHERP) explored provider-level barriers and facilitators to $\mathrm{HCV}$ treatment in a convenience sample of 24 hepatology and primary care providers [2]. The study reported that provider perceptions of specific patient factors such as SUD, reported adherence to medications, and lack of reliable transportation could influence the decision to prescribe HCV treatment. In this issue of Digestive Diseases and Sciences, Patel et al. [3] another group of researchers from the VA CHERP elicited patient-level barriers and facilitators among $40 \mathrm{HCV}$-infected Veterans. The team conducted semi-structured interviews pre- and post-treatment in order to elicit patient perceptions of barriers and facilitators of adherence in the DAA era. Enrolled

Mary Jane Burton

Mary.burton2@va.gov

1 G.V. "Sonny" Montgomery VAMC, 1500 E. Woodrow Wilson Avenue, Jackson, MS, USA patients were predominantly male $(39 ; 98 \%)$ and Caucasian $(28 ; 70 \%)$. One-fourth $(10,25 \%)$ were treatment experienced; more than one-third had cirrhosis $(15 ; 38 \%)$. The majority reported a history of SUD $(32 ; 80 \%)$. National Institute on Drug Abuse (NIDA) quick screens documented current marijuana use, heroin use, and prescription drug misuse in $12(31 \%), 6(15 \%)$, and $4(10 \%)$ of participants, respectively.

The authors utilized the general term "adherence" to signify two overlapping aspects of medication-taking behavior: medication adherence and treatment persistence [4]. Medication adherence refers to the daily compliance with each dose of medication. In this study, medication adherence was determined via patient or provider report of missed doses and/or provider pill counts. Treatment persistence reflects completing the recommended course of therapy; this was determined by investigators' review of provider documentation in the medical record. The author categorized adherence into three groups: took all doses (medication adherence; treatment persistence), missed doses but completed treatment (medication nonadherence; treatment persistence), and incomplete treatment (treatment nonpersistence). Most patients $(n=31)$ took all doses of prescribed medication for the recommended length of therapy. Six patients missed an unspecified number of doses of medication but completed the prescribed course of therapy, and three patients prematurely terminated therapy.

There were no significant differences in baseline characteristics among the three categories of adherence, but the sample size was small. Most patients in the study achieved SVR; treatment failure only occurred in the three patients who did not complete therapy. Characteristics of these three patients were a relatively younger age, $\mathrm{HCV}$ treatment naïve, and lesser liver fibrosis [based on calculated Fibrosis-4 score]. These patients reported higher Patient Reported Outcomes Measurement Information System (PROMIS) $\mathrm{T}$-scores for anxiety, depression, fatigue, and pain than the other enrollees.

In pre-treatment interviews, favorable perceptions of HCV providers were strong motivators for adherence. Patients nearly uniformly identified provider trust and 
satisfaction as treatment facilitators. In post-treatment interviews, provider feedback and positive attributes of the medications influenced compliance. Patients reported that receiving information that the treatment was working, the minimal side effects experienced, and high likelihood of success were motivating factors for adherence.

Before treatment, patients anticipated the following barriers to adherence: medication side effects, forgetting to take medications, and difficulty incorporating medications into their daily routine. After treatment, the lack of side effects became a motivator for completing treatment. Challenges with remembering to take medications and incorporating them into a daily routine remained a perceived barrier. Most patients reported using a system of reminders to assist with medication adherence. The patients who utilized active external reminder systems (e.g., alarm, timer or family member/friend) appeared less likely to miss doses or terminate therapy early as compared to patients who used visual reminders (e.g., calendar). This trend may reflect characteristics of the patients who choose active reminders rather than the efficacy of reminder type. Since the qualitative design did not permit detection of variables that significantly affected medication adherence and treatment persistence, future investigations are warranted in this regard.

Given the rapid evolution of HCV DAA therapy, most patients in the study received drug regimens that are now virtually obsolete. Current DAA regimens have fewer doses and potential side effects than those received by most of the patients in this study. As a result, patients considering $\mathrm{HCV}$ treatment today may be less fearful of medication side effects or of completing an entire course of treatment. Nevertheless, DAA characteristics were only one theme the interviews revealed. The most commonly reported pre-treatment facilitator was provider trust and satisfaction; patients also noted after DAA therapy that reassuring provider feedback motivated treatment completion. Thus, positive patient-provider interactions appear to influence medication adherence and treatment completion.

It is noteworthy that this study was conducted among patients enrolled in the VA Healthcare system. The VA formulary contains all FDA-approved DAAs, and there are currently no restrictions for DAA prescription based on substance use or fibrosis level. Nonetheless, even in a healthcare setting with few systemic-level barriers to DAA treatment, three patients did not complete therapy. To achieve US HCV elimination goals, not only is advocacy needed for policies that promote healthcare access and affordability, but also less tangible aspects of medical care that are equally crucial for HCV elimination must be stressed.

Acknowledgment This work was supported by the Department of Veterans Affairs.

\section{Compliance with Ethical Standards}

Conflict of interest The authors declared that they have no conflict of interest.

\section{References}

1. National Academies of Sciences, Engineering, and Medicine. A national strategy for the elimination of Hepatitis $B$ and C: phase two report. Washington, DC: National Academies Press; 2017.

2. Rogal SS, McCarthy R, Reid A, et al. Primary care and hepatology provider-perceived barriers to and facilitators of Hepatitis C treatment candidacy and adherence. Dig Dis Sci. 2017;62:1933-1943. https://doi.org/10.1007/s10620-017-4608-9

3. Patel K, Zickmund S, Jones H, et al. Determinants of Hepatitis C treatment adherence and treatment completion among veterans in the direct acting antiviral era. Dig Dis Sci. (Epub ahead of print). https://doi.org/10.1007/s10620-019-05590-x.

4. Cramer JA, Roy A, Burell A, et al. Medication compliance and persistence: terminology and definitions. Value Health. $2008 ; 11: 44-47$.

Publisher's Note Springer Nature remains neutral with regard to jurisdictional claims in published maps and institutional affiliations. 